\title{
ON $L^{1}$ ISOMORPHISMS
}

\author{
MICHAEL CAMBERN
}

\begin{abstract}
Let $\left(X_{1}, \Sigma_{1}, \mu_{1}\right)$ and $\left(X_{2}, \Sigma_{2}, \mu_{2}\right)$ be two $\sigma$-finite measure spaces. We show that any isomorphism $T$ of the Banach space $L^{1}\left(X_{1}, \Sigma_{1}, \mu_{1}\right)$ onto the Banach space $L^{1}\left(X_{2}, \Sigma_{2}, \mu_{2}\right)$ which satisfies $\|T\|\left\|T^{-1}\right\|<2$ induces a transformation of the underlying measure spaces.
\end{abstract}

In [1] and [2] it has been shown by D. Amir and M. Cambern that if $Y_{1}$ and $Y_{2}$ are compact Hausdorff spaces, and if there exists an isomorphism $T$ of $C\left(Y_{1}\right)$ onto $C\left(Y_{2}\right)$ with $\|T\|\left\|T^{-1}\right\|<2$, then $Y_{1}$ and $Y_{2}$ are homeomorphic. In this note, we use this theorem to prove an analogous result for $L^{1}$ spaces. (Concerning the terminology "regular set isomorphism" as it is used in this paper, the reader is referred to [6].)

Theorem. Let $\left(X_{1}, \Sigma_{1}, \mu_{1}\right)$ and $\left(X_{2}, \Sigma_{2}, \mu_{2}\right)$ be $\sigma$-finite measure spaces. If there exists an isomorphism $T$ of $L^{1}\left(X_{1}, \Sigma_{1}, \mu_{1}\right)$ onto $L^{1}\left(X_{2}, \Sigma_{2}, \mu_{2}\right)$ satisfying $\|T\|\left\|T^{-1}\right\|$ $<2$, then there exists a regular set isomorphism $\Phi$ of $\left(X_{1}, \Sigma_{1}, \mu_{1}\right)$ onto $\left(X_{2}, \Sigma_{2}, \mu_{2}\right)$.

Proof. Since the measure spaces are $\sigma$-finite, the dual space of $L^{1}\left(X_{i}, \Sigma_{i}, \mu_{i}\right)$ is $L^{\infty}\left(X_{i}, \Sigma_{i}, \mu_{i}\right), i=1,2$ [4, p. 289]. Hence the adjoint transformation $T^{*}$ is an isomorphism of $L^{\infty}\left(X_{2}, \Sigma_{2}, \mu_{2}\right)$ onto $L^{\infty}\left(X_{1}, \Sigma_{1}, \mu_{1}\right)$ satisfying $\left\|T^{*}\right\|\left\|T^{*-1}\right\|<2$. Now $L^{\infty}\left(X_{i}, \Sigma_{i}, \mu_{i}\right)$ is isometrically isomorphic to $C\left(Y_{i}\right), i=1,2$, under the map $\rho_{i}(f)=\hat{f}$, where $Y_{i}$ is the maximal ideal space of $L^{\infty}\left(X_{i}, \Sigma_{i}, \mu_{i}\right)$ and $\rho_{i}$ is the Gelfand representation of $L^{\infty}\left(X_{i}, \Sigma_{i}, \mu_{i}\right),([4$, p. 445] or [5, p. 17]). Define a map $R$ of $C\left(Y_{2}\right)$ to $C\left(Y_{1}\right)$ by $R(\hat{f})=\rho_{1} \circ T^{*} \circ \rho_{2}^{-1}(\hat{f})$, for $\hat{f} \in C\left(Y_{2}\right)$. Then clearly $R$ is an isomorphism of $C\left(Y_{2}\right)$ onto $C\left(Y_{1}\right)$ with $\|R\|\left\|R^{-1}\right\|<2$.

It thus follows that there exists a homeomorphism $\tau$ mapping $Y_{1}$ onto $Y_{2}$. And, being a homeomorphism, $\tau$ carries the clopen sets of $Y_{1}$ onto the clopen sets of $Y_{2}$. Now if $A_{i} \in \Sigma_{i}$, then $\hat{\chi}_{A_{i}}$ is the characteristic function of a clopen subset $U_{A_{i}}$ of $Y_{i}$, and every clopen subset $U$ of $Y_{i}$ is of the form $U_{A_{i}}$, for some $A_{i} \in \Sigma_{i}, i=1,2[5, \mathrm{p}$. 17]. Let $\Phi$ be the map from $\Sigma_{1}$ to $\Sigma_{2}$, defined modulo null sets by $\Phi\left(A_{1}\right)=A_{2}$ if $\tau\left(U_{A_{1}}\right)=U_{A_{2}}$, where $U_{A_{i}} \subseteq Y_{i}$ and is related to $A_{i} \in \Sigma_{i}$ as in the previous sentence. If $\Re_{i}$ denotes the family of null sets in $\Sigma_{i}$, then for $i=1,2, \Sigma_{i} / \Re_{i}$ is isomorphic as a Boolean algebra with the clopen subsets of $Y_{i}$, under the correspondence $A_{i} \leftrightarrow U_{A_{i}}$. Moreover, the Boolean supremum of a sequence $U_{A_{i}}$ of clopen sets is the topological closure of the point set union of the $U_{A_{i}}$. Then, since the homeomorphism $\tau$ of $Y_{1}$ onto $Y_{2}$ preserves both point set unions and topological closures, it clearly effects an order isomorphism between the Boolean algebras of clopen sets

Received by the editors December 7, 1978 and, in revised form, March 13, 1979. AMS (MOS) subject classifications (1970). Primary 46E30, 46E15. 
of the $Y_{i}$, from which it readily follows that the map $\Phi$ of the previous paragraph is a regular set isomorphism.

Remarks AND Problems. (a) The condition $\|T\|\left\|T^{-1}\right\|<2$ in our theorem cannot be removed to allow for arbitrary isomorphisms of $L^{1}\left(X_{1}, \Sigma_{1}, \mu_{1}\right)$ onto $L^{1}\left(X_{2}, \Sigma_{2}, \mu_{2}\right)$ as the following example shows. Let $\left(X_{1}, \Sigma_{1}, \mu_{1}\right)$ be the measure space where $X_{1}=[0,1], \Sigma_{1}$ is the $\sigma$-field of Lebesgue subsets of $[0,1]$ and $\mu_{1}$ is Lebesgue measure. Let $\left(X_{2}, \Sigma_{2}, \mu_{2}\right)$ be defined as follows: $X_{2}=[0,1] \cup\{2\}, \Sigma_{2}$ consists of the Lebesgue measurable subsets of $X_{2}$, and $\mu_{2}$ is the sum of Lebesgue measure on $\Sigma_{2}$ and of the unit point mass concentrated at 2. For each $k=$ $0,1,2, \ldots$, let $I_{k}$ be the subset of $[0,1]$ defined by $I_{k}=\left[\left(2^{k}-1\right) / 2^{k},\left(2^{k+1}-\right.\right.$ 1) $\left./ 2^{k+1}\right)$. We define a map $T$ from $L^{1}\left(X_{1}, \Sigma_{1}, \mu_{1}\right)$ to $L^{1}\left(X_{2}, \Sigma_{2}, \mu_{2}\right)$ by

$$
(T(f))(2)=\int_{I_{0}} f(t) d t
$$

and

$$
(T(f))(t)=f(t)-2^{k+1} \int_{I_{k}} f(t) d t+2^{k+1} \int_{I_{k}+1} f(t) d t
$$

for $f \in L^{1}\left(X_{1}, \Sigma_{1}, \mu_{1}\right)$ and $t \in I_{k}$. $(T(f)$ has not been defined at 1 , but since we are actually defining a map of equivalence classes rather than functions, the value of $(T(f))(1)$ is of no concern.) It is clear that $T$ is linear. It is moreover one-one and surjective since given $g \in L^{1}\left(X_{2}, \Sigma_{2}, \mu_{2}\right)$, the element $f \in L^{1}\left(X_{1}, \Sigma_{1}, \mu_{1}\right)$ defined by

$$
f(t)=g(t)-2 \int_{I_{0}} g(t) d t+2 \cdot g(2)
$$

for $t \in I_{0}$, and

$$
f(t)=g(t)-2^{k+1} \int_{I_{k}} g(t) d t+2^{k+1} \int_{I_{k-1}} g(t) d t
$$

for $t \in I_{k}, k>0$, is such that $T(f)=g$. Thus $T$ is a continuous isomorphism of $L^{1}\left(X_{1}, \Sigma_{1}, \mu_{1}\right)$ onto $L^{1}\left(X_{2}, \Sigma_{2}, \mu_{2}\right)$. However, since $\left(X_{2}, \Sigma_{2}, \mu_{2}\right)$ contains an atom while $\left(X_{1}, \Sigma_{1}, \mu_{1}\right)$ does not, there can exist no regular set isomorphism of $\left(X_{1}, \Sigma_{1}, \mu_{1}\right)$ onto $\left(X_{2}, \Sigma_{2}, \mu_{2}\right)$.

(b) It is known (see [3]) that for the theorem mentioned in the first paragraph of this article, 2 can be replaced by no larger number in the condition $\|T\|\left\|T^{-1}\right\|<$ 2. Is 2 also the "best" number for a theorem of the type obtained in this paper?

(c) Can a theorem analogous to the one of this article be established for $L^{p}$, $1<p<\infty, p \neq 2$ ?

\section{REFERENCES}

1. D. Amir, On isomorphisms of continuous function spaces, Israel J. Math. 3 (1965), 205-210.

2. M. Cambern, On isomorphisms with small bound, Proc. Amer. Math. Soc. 18 (1967), 1062-1066.

3. H. B. Cohen, $A$ bound-two isomorphism between $C(X)$ Banach spaces, Proc. Amer. Math. Soc. 50 (1975), 215-217.

4. N. Dunford and J. T. Schwartz, Linear operators. I, Interscience, New York, 1958.

5. T. Gamelin, Uniform algebras, Prentice-Hall, Englewood Cliffs, N.J., 1969.

6. J. Lamperti, On the isometries of certain function spaces, Pacific J. Math. 8 (1958), 459-466.

Department of Mathematics, University of California, Santa Barbara, California 93106 